\title{
Discriminação racial e vulnerabilidade às DST/Aids: um estudo com adolescentes negras
}

| ${ }^{1}$ Stella R. Taquette, ${ }^{2}$ Zilah Vieira Meirelles |

Resumo: O estudo teve como objetivo verificar a discriminação racial vivenciada por adolescentes negras moradoras em favelas da cidade do Rio de Janeiro e sua possível influência no processo de vulnerabilização ao HIV/ Aids. Utilizou-se uma combinação de métodos, quantitativo e qualitativo. Este artigo se refere a um recorte da etapa qualitativa desenvolvida por meio de dez grupos focais com a participação de 139 adolescentes. Seguiu-se um roteiro para o debate contendo dois grupos temáticos: sexualidade/ DST/Aids/gênero e raça/cor/discriminação. Os relatos foram gravados e o material transcrito organizado conforme os temas tratados e analisados criticamente por equipe multidisciplinar. Os dados coletados foram classificados em categorias específicas articuladas aos pressupostos teóricos, a fim de responder às questões formuladas, tendo por base os objetivos da pesquisa. Os resultados revelaram que as adolescentes negras sofrem discriminação racial no seu cotidiano, que é manifestada nas expressões de suas falas, referindo-se ao aspecto físico, ao caráter e à capacidade intelectual. Tais condutas discriminatórias dificultam o acesso aos serviços de saúde e induzem um atendimento de baixa qualidade. Concluiu-se que a discriminação racial vivida por estas adolescentes negras, na cidade do Rio de Janeiro, influencia o desenvolvimento da autoestima e contribui para a construção de uma identidade negativa que, aliada ao racismo e à pobreza, se configura num contexto de vulnerabilidade às DST/Aids. Sugere-se que estes dados sejam levados em consideração na elaboração de políticas públicas para que ofereçam atenção diferenciada àqueles que estão inseridos de forma desigual na sociedade.

1 Professora associada da Faculdade de Ciências Médicas (FCM) da Universidade do Estado do Rio de Janeiro (UERJ) Endereço eletrônico: stella. taquette@gmail.com

${ }^{2}$ Núcleo de Estudos da Saúde do Adolescente (NESA) da UERJ. Endereço eletrônico: zmeirelles@terra.com.br 
A epidemia da Aids no Brasil vem apresentando tendência de feminização, pauperização e heterossexualização. Esses fatos trouxeram novos desafios às autoridades sanitárias, em especial no que diz respeito às mulheres, que estão nos mais baixos patamares da escala social e são, em sua maioria, da raça negra. Desde o ano 2000, quando a raça/cor passou a ser informada nas fichas de notificação, houve acréscimo do número de casos de Aids entre pretos e pardos (BRASIL/MS, 2007). A complexidade da epidemia em mulheres coloca em foco as relações desiguais entre os gêneros e reforça a necessidade de se analisar e abordar a violência de gênero contra as mulheres e a vulnerabilidade à infecção pelo HIV e outras DST, de maneira inter-relacionada (VILELA, 2010).

A epidemia da Aids tem se mostrado dinâmica e volátil. No início da epidemia no Brasil, utilizava-se o conceito de "grupo de risco" que posteriormente foi sendo substituído pelo de "comportamento de risco". As vítimas atuais, em especial as mulheres negras e pobres, não pertencem a nenhum grupo de risco, nem tampouco apresentam comportamento de risco, mas vivem em contextos sociais onde vários fatores potencializam a vulnerabilidade às DST/Aids, entre eles a violência baseada em gênero, a discriminação racial, a pobreza e a baixa escolaridade (SANCHEZ, 2007; SEFFNER, 2009). A feminização da epidemia de Aids e outras DST é resultante da convergência desses fatores, o que torna complexa sua análise, que deve levar em consideração também a violência doméstica e sexual, o estigma e violação dos direitos humanos e o não-reconhecimento das adolescentes e jovens como sujeitos de direitos (VILELA, 2012).

$\mathrm{O}$ conceito de vulnerabilidade é norteador da resposta nacional à Aids e pressupõe, nesta perspectiva, a compreensão de que a epidemia resulta de um conjunto de fatores individuais, sociais e programáticos que incidem diretamente sobre a maior ou menor exposição de homens e mulheres ao HIV/Aids (BRASIL/ MS, 2010). Compreender estes contextos de vulnerabilidade tem sido necessário nas estratégias de enfrentamento da epidemia (MAN, 1993; BERKMAN, 2005; AYRES, 2006; PAIVA, 2006; SANTOS, 2009). O conceito de vulnerabilidade, segundo Ayres (2003), implica uma percepção ampliada e reflexiva da epidemia que identifica aspectos que vão de susceptibilidades orgânicas à forma de estruturação de programas de saúde, passando por aspectos comportamentais, culturais, econômicos e políticos (BLACKWELL, 2002; CARNEIRO, 2002). 
As mulheres negras, em sua maioria, estão situadas nos mais baixos patamares

de renda, evidenciando uma possível relação entre raça/cor na distribuição da riqueza (LOPES, 2005). Os indicadores de saúde da população revelam que as mulheres negras apresentam maior razão de mortalidade, sofrem duas vezes mais agressão física na gravidez e têm maior taxa de hipertensão arterial, diabetes, tabagismo e sífilis (MARTINS, 2006; CAVALCANTE et al., 2006).

Em relação às adolescentes, destaca-se que são as vítimas preferenciais de abuso sexual e de exploração sexual comercial (ABRAPIA, 2008). Têm risco aumentado de infecções genitais, devido à maior exposição do epitélio uterino, e estão numa posição de menor poder de negociação de práticas sexuais seguras, em consequência da desigualdade de gênero, além de não terem reconhecida a legitimidade de seu exercício sexual (TAQUETTE, 2009). Frequentemente lhes é negado atendimento nos serviços de saúde quando não acompanhadas de seus responsáveis, impedindo as ações de prevenção e tratamento de DST, essenciais para deter o avanço do HIV.

A associação entre pobreza/violência/sexo feminino, fatores que potencializam os contextos de vulnerabilidades às DST/Aids, também foi verificada em estudos realizados com adolescentes e jovens (TAQUETTE et al., 2003; 2004). Por outro lado, outros autores questionam a associação entre raça/cor e vulnerabilidade às DST/Aids devido à insuficiência de dados epidemiológicos disponíveis (FRY et al., 2007).

Em síntese, há argumentos que evidenciam uma maior exposição das mulheres negras a fatores que podem potencializar a susceptibilidade à infecção pelo HIV, entre eles: os individuais (baixa autoestima, atividade sexual precoce, não adoção de práticas sexuais seguras), os sociais (discriminação racial, menor acesso à renda/ emprego/educação/ saúde, violência sexual/gênero) e os coletivos/programáticos (racismo institucional, ausência de políticas públicas direcionadas a este público, etc.).

Neste estudo, procura-se abordar as desigualdades de gênero e étnico-raciais que são fatores que vêm contribuindo com a vulnerabilidade ao HIV/Aids. Para tanto, objetivou-se identificar a vivência da discriminação racial de mulheres adolescentes, moradoras de favelas da cidade do Rio de Janeiro, através de suas formas de expressão e influência no processo de agravamento do HIV/Aids. 


\section{População e método}

$\mathrm{Na}$ primeira etapa, quantitativa, realizou-se estudo transversal com amostra representativa de adolescentes do sexo feminino $(\mathrm{n}=816)$, calculada segundo o censo demográfico do IBGE (2000), moradoras de dez favelas do Rio de Janeiro localizadas em bairros variados, abrangendo as cinco áreas de planejamento (AP) da cidade. Devido à violência existente nas favelas da cidade, o estudo só pôde ser realizado naquelas onde já havia um vínculo prévio com a equipe de pesquisadores. As adolescentes foram convidadas a participar da pesquisa em seus locais de convivência e com a intermediação de lideranças locais. Portanto, a escolha das favelas não foi aleatória. Foram realizadas entrevistas para o conhecimento do perfil sociodemográfico e sexual das adolescentes e verificação da incidência de DST/Aids por meio de exames clínicos e laboratoriais (CLAP/ OPS/OMS, 1995).

A etapa qualitativa, a que se refere este artigo, foi realizada através de dez grupos focais (um em cada favela) com as adolescentes que participaram da fase quantitativa e aceitaram o convite para compor os grupos. Estes foram compostos por no máximo 15 adolescentes cada, independentemente de idade ou classificação racial, e foi utilizado um roteiro na condução da discussão com os temas sexualidade/DST/Aids/gênero e raça/cor/discriminação. Participaram, no total dos dez grupos focais, 139 adolescentes do sexo feminino. As reuniōes foram gravadas e, através de leitura e releitura do material transcrito, uma préanálise identificou as principais variáveis temáticas. Em seguida, estabeleceram-se interrogaçôes para identificar o que surgiu de relevante e serem criadas categorias específicas. Ao final, os dados foram articulados aos pressupostos teóricos da pesquisa para responder às questôes da mesma com base em seus objetivos. A interpretação dos dados foi feita em equipe multidisciplinar tendo como base teórico-conceitual a hermenêutica-dialética (MINAYO, 2001; 2006). O estudo foi previamente aprovado pelo Comitê de Ética em Pesquisa da Universidade do Estado do Rio de Janeiro.

\section{Resultados e Discussão}

Cerca de três quartos (74\%) das adolescentes entrevistadas, segundo dados da fase quantitativa do estudo, se autodeclararam pretas ou pardas, e essa proporção se manteve nos grupos focais realizados. As discussões em cada grupo 
duraram em média 53 minutos. A primeira pergunta norteadora do debate sobre discriminação racial procurou conhecer a opinião das adolescentes sobre como a sociedade vê uma jovem negra e se elas sofrem algum constrangimento na sociedade devido a sua raça.

Em seguida, foi indagado às participantes se as mulheres jovens e adolescentes preferem homens negros ou brancos para namorar e terem filhos. Por último, (3) foi investigado o conhecimento delas sobre DST/Aids e a relação destas doenças com a população negra e o acesso aos serviços de saúde.

As falas das adolescentes deram origem a quatro categorias empíricas que se articulam entre si: (I) - Expressões sociais de discriminação; (II) - Manifestações de sofrimento provocado pela discriminação; (III) - Relação entre raça/cor e sexualidade/DST/Aids; (IV) - Dificuldade de acesso a serviços e racismo institucional.

$\mathrm{Na}$ primeira categoria, "expressóes sociais de discriminação", estas foram identificadas nos relatos baseadas em três diferentes manifestaçôes: as advindas da aparência física; as decorrentes de questôes de caráter e outras referentes à capacidade intelectual. Nas manifestações baseadas na "aparência física", as adolescentes negras referiram ser qualificadas como feias, sujas e fedorentas, segundo se apurou por ocasião da interpretação dos dados colhidos, com as seguintes expressōes:

Miquinha [...] aquela macaca do cabelo duro!

Quando ela vai à praia falam que ela é porca...

A criança branca, mesmo sujinha, é bonitinha.

Você tá no ônibus e alguém levanta o braço, se você sentir um cheiro de suor, olham logo pra pessoa escura!

As pessoas acham que o negro é sempre fedorento...

A gente foi no zoológico e quando estávamos perto dos macacos, o pessoal falou: preto, aí teus parentes.

Nas falas compreendidas na categoria de "ausência de caráter", as adolescentes situaram as diferenças com as seguintes frases:

A gente entra numa loja o segurança vem logo atrás da gente.

O branco no volante é doutor; preto no volante é ladrão.

Tem um ditado que é assim: preto quando corre é bandido.

Preto na praia é pra fazer arrastão...

Viu um pretinho, já guarda a bolsa, esconde o dinheiro! 
No que se refere à questão da "incapacidade intelectual", não podia ser diferente. As adolescentes pontuaram a incapacidade com as seguintes expressōes: Eles acha que nois... da cor assim, num tem a mesma capacidade dum branco... (sic) Às vezes o negro é melhor do que muito branco, mas eles num dão oportunidade, por serem negras e do morro, entendeu? (sic)

Mesma coisa é com concurso... Muitas pessoas acham que negro não vai conseguir passar.

Os exemplos acima evidenciam a existência de estereótipos negativos e o tratamento desigual que ocorre entre brancos e negros. Representam uma barreia à ascensão social dos negros e contribuem para manter a distância social entre eles e os brancos. Essa discriminação é vivenciada cotidianamente. Pode ser percebida por meio de sinais sutis, como vimos neste estudo e é corroborada por Oliveira et al. (2003), ao citarem o modo como "normalmente" a população negra é tratada, por exemplo, na aspereza de um atendente numa loja, no olhar aguçado e desconfiado de um segurança, por um taxista que não para ao ser chamado.

Outros estudos evidenciam que o insulto racial leva a uma identidade social estigmatizada, como um inferior racial (GUIMARÃES, 2000). Tanto as vítimas dos insultos, quanto seus perpetradores aceitam tais estereótipos como naturais, que se cristalizam em crenças de que são inferiores e assim se constrói a institucionalização da inferioridade racial, evidenciado nos sentimentos das adolescentes, de que são sujas, têm cabelo ruim, são vistas potencialmente como ladras e incompetentes. Essa visão que se tem da população negra é, segundo Santos (2002), a base da ideologia racista, em que o visível define a concepção que se tem do "ser negro". O racismo é aparentemente velado por uma polidez superficial revestida de comportamentos discriminatórios expressados por meio de piadas, brincadeiras, ditos populares (LIMA, 2004).

A segunda categoria, "manifestação do sofrimento provocado pela discriminação" verificou-se que as dificuldades enfrentadas pelas adolescentes, bem como as manifestações de que desejam "clarear a família", por meio do casamento com homens brancos explicitam o sofrimento resultante da discriminação e a busca incessante de serem mais bem vistas socialmente. Nesta categoria compreendemse as seguintes falas:

Eu sou preta e não gosto de homem mais preto que eu não!

Ah, eu gosto dum branquinho... 
Eu vou falar a verdade: eu prefiro namorar gente clara, assim moreninho porque, quando eu tiver um filho, o meu filho não fique de cabelo duro [...] cabelo duro é uma tristeza...

Minha mãe fala: você tem que arrumar um namorado branquinho pra clarear a família!

Nos debates dos grupos focais, muitas adolescentes expressaram seu descontentamento com a própria raça, tentando mudar a imagem mediante processo de branqueamento, como tingimento, alisamento e alongamento do cabelo, assemelhando-se às mulheres brancas. As adolescentes afirmaram que se sentem feias e discriminadas, inclusive nas suas próprias famílias. Algumas moças relataram que mordem os lábios na intenção de reduzir seu tamanho e colocam pregador de roupa no nariz para afiná-lo, assim como evitam comer feijão para não ficarem mais pretas. As adolescentes negras renegam a própria cor porque ela está relacionada só a coisas ruins, tais como ser feia, suja, fedorenta, do cabelo duro, "beiçuda", ladra e incapaz. Consequentemente, para não sofrerem e serem aceitas, sobretudo pelos homens, "querem ser brancas".

Esta fase da vida, juntamente com a infância, é crucial no desenvolvimento da identidade e da autoestima, e proporciona a competência emocional necessária para se enfrentar as dificuldades inerentes ao viver. Bennet (2010), em estudo realizado com mulheres de classes sociais de menor renda, demonstrou que a percepção crônica e cotidiana de discriminação está associada a sintomas depressivos. Ser construído em um ambiente social e familiar que coloca as pessoas de cor preta numa posição menor, de inferioridade e de incapacidade, contribui para a crença de que estas não têm os mesmos direitos humanos dos brancos. Essa "inferioridade" dificulta que a população negra atinja patamares mais altos na escala social e intelectual. Os indivíduos negros acabam por se submeter, sem revolta, a serem tratados de forma pior do que os brancos, não tendo as mesmas oportunidades de conquistas sociais, assim como aceitam não ter seus direitos de saúde garantidos e, consequentemente, ficam mais expostos às doenças, com repercussões na saúde física e psicológica.

A terceira categoria, "relação entre a raça/cor e a sexualidade/DST/Aids", surge através de um número significativo de relatos sugestivos da associação entre a raça e o risco de gravidez e de DST/Aids, como se pode constatar:

Ih, a negrinha já tá grávida...

[...] cabelo duro, pro alto, só vive prenha, esses papos! 
Preta, pobre, só sabe ir pro pagode e rebolar, fica grávida e depois não trabalha...

Ah, você é preta, você tem doença! Ainda mais se você mora no morro!

As DST já tá relacionado à mulher negra (sic).

Principalmente a Aids, que dizem que veio da África...

A relação entre raça/cor e sexualidade externada nas falas das adolescentes estudadas pode estar refletida no pensamento de que os negros têm maior vulnerabilidade, de que Aids é originária da África e de que as mulheres têm mais atividade sexual, pois engravidam e adoecem mais. Outro aspecto que contribui para esta associação é o início da atividade sexual com mais baixa idade neste segmento populacional e a lógica de gênero, que dificulta a negociação quanto ao uso de preservativos, colocando as adolescentes numa posição mais vulnerável às DST/Aids.

A vulnerabilidade das mulheres ao HIV/Aids foi documentada por Lopes et al. (2007) em estudo multicêntrico envolvendo 1.068 mulheres vivendo com HIV. Foram verificadas associações estatisticamente significativas entre negros e não-negros em relação a: escolaridade, renda mensal, oportunidade de serem atendidas por profissionais de saúde e via sexual de exposição.

Apesar de alguns autores (MAIO et al., 2005) questionarem a veiculação da raça negra ao aumento da vulnerabilidade ao HIV/Aids, o fato de essa epidemia estar atingindo as populações mais pobres torna imprescindível a valorização da questão da discriminação racial como fator fundamental para a manutenção das mesmas nos patamares mais baixos da hierarquia social e também do aumento progressivo do número de negros acometidos pela Aids (BRASIL/MS, 2009).

A quarta categoria empírica, "dificuldade de acesso a serviços e racismo institucional", emerge das queixas das entrevistadas sobre a falta de serviços de saúde disponíveis, pois os existentes são distantes de seus locais de moradia, exigem filas, deslocamento de suas casas de madrugada para pegar a distribuição de senhas, além de longo tempo de espera para o atendimento. As adolescentes, que não conseguem ser consultadas por falta de vagas, retornam às suas casas exaustas e frustradas por terem gasto dinheiro de passagem inutilmente. Frequentemente, desistem de retornar ao serviço de saúde e procuram resolver seus problemas recorrendo a alternativas como balcôes de farmácia, remédios de terceiros e práticas caseiras. Relataram, ainda, situações de discriminação perpetrada por profissionais de saúde, por meio de ofensas e atendimento de 
má qualidade. O não-acesso aos serviços de saúde resulta na impossibilidade de tratamento adequado das infecções sexualmente transmissíveis, de aquisição de insumos de prevenção e de orientação em prevenção de DST/Aids.

As adolescentes reproduziram, com as seguintes frases, as dificuldades que enfrentam nos serviços,:

Pra você conseguir ir num Posto de Saúde, tem que chegar de madrugada... você fica lá esperando... aí quando vai dar o número: não tem mais vaga, não tem médico...

É só chegar uma preta que: oh, já vem essas faveladas...

O médico não atende a gente direito, porque acha que a gente tá fedendo...

No ambiente pesquisado, além da escassa oferta de serviços de saúde para a população moradora de favela, nos locais onde são atendidas, segundo os relatos das adolescentes, frequentemente são vítimas de racismo institucional, aqui considerado como o fracasso da instituição em prover um serviço apropriado às pessoas por causa da sua raça/cor. Esta é uma das principais barreiras de acesso da população negra à saúde, manifestando-se por meio de preconceitos involuntários, ignorância, negligência e estereótipos racistas, o que acaba por afastar os usuários desses serviços (KALCKMANN et al., 2007).

Cabe salientar que o racismo é o fenômeno ideológico caracterizado pela atribuição de características negativas a determinados padrões de diversidade e significados sociais negativos aos grupos que os detêm. Reafirma-se no dia a dia pela linguagem comum, mantém-se e alimenta-se pela tradição e pela cultura, influencia a vida, as relaçôes estabelecidas entre as pessoas e também a organização e o funcionamento das instituições. Portanto, a discriminação vem adicionar mais um fator de vulnerabilidade ao contexto de pobreza e de violência de gênero vivido pela maioria das mulheres negras, pois as impede de terem acesso a tratamento preventivo adequado, aumentando o risco de serem portadoras de infecções genitais não diagnosticadas e não tratadas. Ou seja, a própria estrutura organizacional da saúde expele este segmento populacional ao atendimento, já tão precário, sendo este mais dos fatores que constitui a vulnerabilidade ao HIV/Aids. Nesse sentido, as ações integradas para a promoção da equidade de gênero e em saúde e para o enfrentamento da epidemia de HIV/DST entre as mulheres devem ser construídas levando em consideração a complexidade e a multidimensionalidade das vulnerabilidades, em que se inclui o racismo, as desigualdades étnicas e raciais e a discriminação racial. 
Estudo sobre classificação racial realizado por Bastos (2008) verificou dados semelhantes ao desta pesquisa na existência de uma tendência de branqueamento da população das camadas sociais privilegiadas. Muitas pessoas que são pardas ou pretas são classificadas por entrevistadores como brancos. Estes dados nos levam a crer que embora na sociedade brasileira não exista uma segregação racial legal, o significado social negativo atribuído a determinados padrões fenotípicos resulta em um tratamento desigual e em uma enorme barreira que impede e dificulta a mobilidade social da população negra.

É no convívio social que as crianças aprendem e internalizam o que se veicula no contexto em que vivem. Portanto, quando são discriminadas pela cor/raça, as representações racistas e o racismo são aprendidos, internalizados e propagados intra e intergerações. A maioria não consegue resistir a isso e renega a sua raça, abandonando sua tradição cultural ou étnica e passando a ter postura e atitude de brancos para ser bem-sucedida e poder ascender socialmente.

\section{Conclusões}

O estudo evidencia a complexidade da epidemia do HIV/Aids em mulheres jovens. Seus resultados colocam em foco que as relações desiguais entre os gêneros, as disparidades socioeconômicas e as discriminações étnico-raciais contribuem para o aumento da suscetibilidade deste grupo populacional ao HIV/Aids. Todavia, suas abordagens e intervenções devem ser realizadas de maneira inter-relacionada.

As narrativas dessas adolescentes expressam vivências de preconceito e discriminação que as levam ter uma autoimagem distorcida, provocando sentimentos de insegurança quanto ao aspecto físico, ao caráter e à capacidade intelectual. Soma-se a este quadro o racismo institucional que as vitimiza nos serviços de saúde, reforçado pela ausência do autocuidado mesmo diante do fato de se perceberem com maior chance de engravidar e adquirir doença sexualmente transmissível e Aids.

Para este público de adolescentes do sexo feminino, moradoras de espaços populares (favelas), sugere-se maior incremento de ações intra e intersetoriais, bem como dos trabalhos que vêm sendo realizados pelo Ministério da Saúde, na área de promoção aos direitos sexuais e reprodutivos, diagnóstico, assistência, prevenção e tratamento de DST/HIV/Aids e apoio às mulheres que vivem com HIV e Aids. Sugere-se, também, a implementação de políticas específicas para a 
juventude, visando superar as situações de vulnerabilidade frente ao HIV/Aids.

Cabe ressaltar que o combate à pobreza entre as mulheres é condição essencial para o desenvolvimento social, econômico, cultural e ambiental e, ainda, para o controle da epidemia de Aids e outras DST.

As conclusões desta pesquisa não podem ser generalizadas para a cidade do Rio de Janeiro como um todo, mas, ainda assim, é possível fazer recomendações para as políticas públicas a partir delas, que poderão trazer contribuições ao denso estrato populacional de adolescentes do sexo feminino que residem nas favelas cariocas. ${ }^{1}$

\section{Agradecimentos}

Agradecemos a valiosa contribuição de Clátia Regina Vieira, advogada e pesquisadora do Instituto de Pesquisas Afro-brasileiras do Rio de Janeiro, na coleta de dados e interpretação dos resultados da pesquisa.

\section{Referências}

ASSOCIAÇÃO BRASILEIRA MULTIPROFISSIONAL de Proteção à Infância e à Adolescência. Abuso sexual de crianças e adolescentes. Disponível em: <http://www. observatoriodainfancia.com.br>. Acesso em: 08 mar 2008.

AYRES, J.R.C.M. et al. Vulnerability, human rights and comprehensive health care needs of young people living with HIV/Aids. American Journal of Public Health, v. 96, n.6, p. 1001-1006, 2006.

AYRES, J.R.C.M. et al. O conceito de vulnerabilidade e as práticas de Saúde: novas perspectivas e desafios. In: CZERESNIA, D. (Org.). Promoção da saúde: conceitos, reflexões, tendência. Rio de Janeiro, Fiocruz, 2003. p. 117-139.

BASTOS, J.L. et al. Diferenças sócio-econômicas entre autoclassificação e heteroclassificação de cor/raça. Revista de Saúde Pública. São Paulo, v.42, n. 2, p. 324-334, 2008.

BENNET, I.M. et al. Perceived discrimination and depressive symptoms, smoking, and recent alcohol use in pregnancy. Birth, v. 37, n. 2, p. 90-97, 2010.

BERKMAN, A. et al. A critical analysis of the Brazilian response to HIV/Aids: lessons for controlling and mitigation the epidemic in developing countries. American Journal of Public Health, v. 95, p. 1162-72, 2005.

BLACKWELL, M.; NABER, N. Interseccionalidade em uma era de globalização: as implicações da conferência mundial contra o racismo para práticas feministas transnacionais. Estudos Feministas. Florianópolis, v. 10, n. 1, p. 189-198, jan 2002. 
BRASIL. Ministério da Saúde. Boletim Epidemiológico de Aids 2008. Disponível em: <http://www.Aids.gov.br. Acesso em: 07 mar 2009.

BRASIL. Ministério da Saúde. O mapa da Aids no Brasil 2009. Disponível em: <http:// www.Aids.gov.br/data/Pages/LUMISE77B47C8ITEMID0863246E010245EDB538 EB99511F42B2PTBRIE.htm>. Acesso em: 29 nov 2009.

CARNEIRO, S.A. A Batalha de Durban. Estudos Feministas. Florianópolis, v. 10, n. 1, p. 209-214, jan 2002.

CAVALCANTE, L.F.; GOMES, R.; MINAYO, M.C.S. Representações sociais de profissionais de saúde sobre violência sexual contra a mulher: estudo em três maternidades do Rio de Janeiro. Cadernos de Saúde Publica. Rio de Janeiro, v. 22, n. 1, p. 31-39, 2006.

CENTRO LATINOAMERICANO DE PARINATOLOGIA y Desarrollo Humano. Organización Panamericana de la Salud. Sistema Informático Del Adolescente. Montevideo, ago 1995 (Publicación Científica CLAP 1342.01).

FRY, P.H. et al. Aids tem cor ou raça? Interpretação de dados e formulação de políticas de saúde no Brasil. Cadernos de Saúde Pública. Rio de Janeiro, v. 23, n. 3, p. 497-523, 2007.

GUIMARÁES, A.S.A. O insulto racial: as ofensas verbais registradas em queixas de discriminação. Estudos Afro-Asiáticos. Rio de Janeiro, v. 38, p. 31-48, 2000.

INSTITUTO BRASILEIRO DE GEOGRAFIA A ESTATÍSTICA. Censo 2000. Disponível em: <www.ibge.gov.br> Acesso em: 27 mar 2005.

KALCKMANN, S. et al. Racismo institucional: um desafio para a equidade no SUS? Saúde e Sociedade. São Paulo, v. 6, n. 2, p. 146-55, 2007.

LIMA, M. E. Sucesso social, branqueamento e racismo. Psicologia: Teoria e Pesquisa. Brasília, v. 20, n. 1, p. 11-19, 2004.

LOPES, F. Para além das barreiras dos números: desigualdades raciais e saúde. Cadernos de Saúde Pública. Rio de Janeiro, v. 21, n. 5, p. 1.595-1.601, 2005.

LOPES, F.; BUCHALLA, C.M.; AYRES, J.R.C.M. Mulheres afrodescendentes e não afrodescendentes e vulnerabilidade ao HIV-Aids no estado e São Paulo, Brasil. Revista de Saúde Pública. São Paulo, v. 41, supl. 2, p. 39-46, 2007.

MAIO, M.C.; MONTEIRO, S. Tempos de racialização: o caso da "saúde da população afrodescendente" no Brasil. História, Ciência e Saúde - Manguinhos. Rio de Janeiro, v. 12, n. 2, p. 419-46, 2005.

MANN, J. et al. (Orgs.). A Aids no mundo. Rio de Janeiro: Relume Dumará, 1993. (História Social da Aids, 1).

MARTINS, A.L. Mortalidade materna de mulheres afrodescendentes no Brasil. Cadernos de Saúde Pública. Rio de Janeiro, v. 22, n. 11, p. 2473-79, 2006. 
MINAYO, M.C.S. O desafio do conhecimento. Pesquisa qualitativa em saúde. São Paulo: Hucitec, 2006. 407p.

MINAYO, M.C.S.; ASSIS, S.G.; SOUZA, E.R. Avaliação por triangulação de métodos. Rio de Janeiro: Fiocruz, 2006. 244p.

MINAYO, M.C.S. et al. Pesquisa Social. Teoria, método e criatividade. Petrópolis: Vozes, 2001. OLIVEIRA, C.L.P.; BARRETO, P.C.S. Percepção do racismo no Rio de Janeiro. Estudos Afro-Asiáticos. Rio de Janeiro, v. 2, p. 183-213, 2003.

PAIVA, V.; PUPO, L.R.; BARBOZA, R. O direito à prevenção e os desafios da redução da vulnerabilidade ao HIV no Brasil. Revista de Saúde Pública. São Paulo, v. 40, supl., p. 109-119, 2006.

SÁNCHEZ, A.M.; BERTOLOZZI, M.R. Pode o conceito de vulnerabilidade apoiar a construção do conhecimento em Saúde Coletiva? Ciência \& Saúde Coletiva. Rio de Janeiro, v. 12, n. 2, p. 319-324, 2007.

SANTOS, G.A. Selvagens, exóticos, demoníacos. Ideias e Imagens sobre uma gente de cor preta. Estudos Afro-Asiáticos. Rio de Janeiro, v. 2, p. 275-89, 2002.

SANTOS, N.J.S. et al. Violence and health: theoretical, methodological, and ethical contributions from studies on violence against women. Cadernos de Saúde Pública. Rio de Janeiro, v. 25, supl. 2, p. S321-S333, 2009.

SEFFNER, F. O conceito de vulnerabilidade: uma ferramenta útil em seu consultório. Disponível em: <http://www. Aids.gov>. Acesso em: 17 jul 2009.

TAQUETTE, S.R. O paradoxo da moral sexual na adolescência e as DST/Aids. In:

(Org.). Aids e juventude: gênero, classe e raça. Rio de Janeiro: EdUERJ, 2009. p. 135-154.

TAQUETTE, S.R. et al. Relacionamento violento na adolescência e risco de DST/Aids. Cadernos de Saúde Pública. Rio de Janeiro, v. 19, p. 1437-1444, 2003.

TAQUETTE, S.R.; VILHENA, M.M.; PAULA, M.C. Doenças sexualmente transmissíveis e gênero: estudo transversal com adolescentes no Rio de Janeiro. Cadernos de Saúde Pública. Rio de Janeiro, v. 20, n. 1, p. 282-290, 2004.

VILLELA, W. V. Mapeamento da situação do Plano Integrado de Enfrentamento da Feminização da Aids e outras DST. Rio de Janeiro. Disponível em: <http://sistemas.aids. gov.br/feminizacao>. Acesso em: 31 jan 2013.

\section{Nota}

${ }^{1}$ S.R. Taquette foi responsável pelo estudo, participou da coleta, análise e interpretação dos dados e redação do manuscrito. Z.V. Meirelles participou da coleta, interpretação dos dados e redação do manuscrito. 
Racial discrimination and vulnerability to STD/ AIDS: a study of black teenage girls in Rio de Janeiro

This study aimed to verify the racial discrimination experienced by black teenage girls living in shantytowns in Rio de Janeiro city and its possible influence on their vulnerability to HIV/AIDS. We used a combination of quantitative and qualitative methods, based on data collected from focus groups composed of 139 teenage girls. Group discussions followed a script of subjects involving two thematic areas: sexuality/STDs/AIDS/gender and race/color/discrimination. The discussions were recorded and the transcribed texts were critically analyzed by a multidisciplinary team. The data collected were classified in specific categories associated with the theoretical assumptions, to respond to the research questions. The results reveal that the girls suffer racial discrimination in their daily lives, manifested in disparaging remarks and attitudes toward their way of speaking, physical appearance, character and intellectual capacity. This discriminatory behavior hinders their access to health services and also reduces the quality of the services received. The conclusion is that the racial discrimination experienced by these black teenage girls influences their development of self-esteem and contributes to the construction of a negative identity, which allied with poverty creates a context of vulnerability to DSTs/AIDS. We suggest these findings be taken into consideration in the formulation of public policies to offer better health care services to those who suffer from inequality and discrimination.

> Key words: prejudice; racism; teenage health; vulnerability; STD/ AIDS; qualitative research. 